\title{
Astrophysical False Positives Encountered in Wide-Field Transit Searches
}

\author{
David Charbonneau*, Timothy M. Brown ${ }^{\dagger}$, Edward W. Dunham**, \\ David W. Latham ${ }^{\ddagger}$, Dagny L. Looper* and Georgi Mandushev** \\ ${ }^{*}$ California Institute of Technology, MC 105-24, 1200 E. California Blvd., Pasadena, CA 91125 \\ ${ }^{\dagger}$ High Altitude Observatory, Ntl Ctr for Atmospheric Research, P.O. Box 3000, Boulder, CO 80307 \\ ${ }^{* *}$ Lowell Observatory, 1400 W. Mars Hill Road, Flagstaff, AZ 86001 \\ ${ }^{\ddagger}$ Harvard-Smithsonian Ctr for Astrophysics, 60 Garden St., MS-20, Cambridge, MA 02138
}

\begin{abstract}
Wide-field photometric transit surveys for Jupiter-sized planets are inundated by astrophysical false positives, namely systems that contain an eclipsing binary and mimic the desired photometric signature. We discuss several examples of such false alarms. These systems were initially identified as candidates by the PSST instrument at Lowell Observatory. For three of the examples, we present follow-up spectroscopy that demonstrates that these systems consist of (1) an M-dwarf in eclipse in front of a larger star, (2) two main-sequence stars presenting grazing-incidence eclipses, and (3) the blend of an eclipsing binary with the light of a third, brighter star. For an additional candidate, we present multi-color follow-up photometry during a subsequent time of eclipse, which reveals that this candidate consists of a blend of an eclipsing binary and a physically unassociated star. We discuss a couple indicators from publicly-available catalogs that can be used to identify which candidates are likely giant stars, a large source of the contaminants in such surveys.
\end{abstract}

\section{WIDE-FIELD TRANSIT SURVEYS}

More than 20 groups worldwide are now engaged in photometric surveys aimed at detecting Jupiter-sized planets in tight orbits about their parent stars [for a review, see 1,2]. Several of these projects are small, automated systems with modest apertures (typically $10 \mathrm{~cm}$ ) and CCD cameras (typically $2 \mathrm{k} \times 2 \mathrm{k}$ arrays of $\sim 15 \mu \mathrm{m}$ pixels), which monitor several thousand stars simultaneously in a very wide field-of-view $\left(6^{\circ}-9^{\circ}\right.$ square). Examples of such instruments are STARE ${ }^{1}$ [3, located on Tenerife], PSST [located in northern Arizona], Sleuth ${ }^{2}$ [4, located in southern California], Vulcan ${ }^{3}[5,6$, located in central California] and Vulcan South ${ }^{4}$ [to be located at the South Pole], HAT ${ }^{5}$ [7, located in southern Arizona], and SuperWASP ${ }^{6}$ [8, located on La Palma]. The advantage that all such projects offer over deeper transit surveys is the brightness of the target stars (typically $8 \leq V \leq 13$ ), which facilitates radial velocity measurements aimed at detecting

${ }^{1}$ http://www.hao.ucar.edu/public/research/stare/stare.html

2 http://www.astro. caltech.edu/ ftod/sleuth.html

3 http://web99.arc.nasa.gov/ vulcan/

4 http://web99.arc.nasa.gov/ vulcan/south

5 http: //cfa-www.harvard.edu/ gbakos/HAT/

6 http://www. superwasp.org/

CP713, The Search for Other Worlds: Fourteenth Astrophysics Conference, edited by S. S. Holt and D. Demings

(C) 2004 American Institute of Physics 0-7354-0190-X/04/\$22.00 

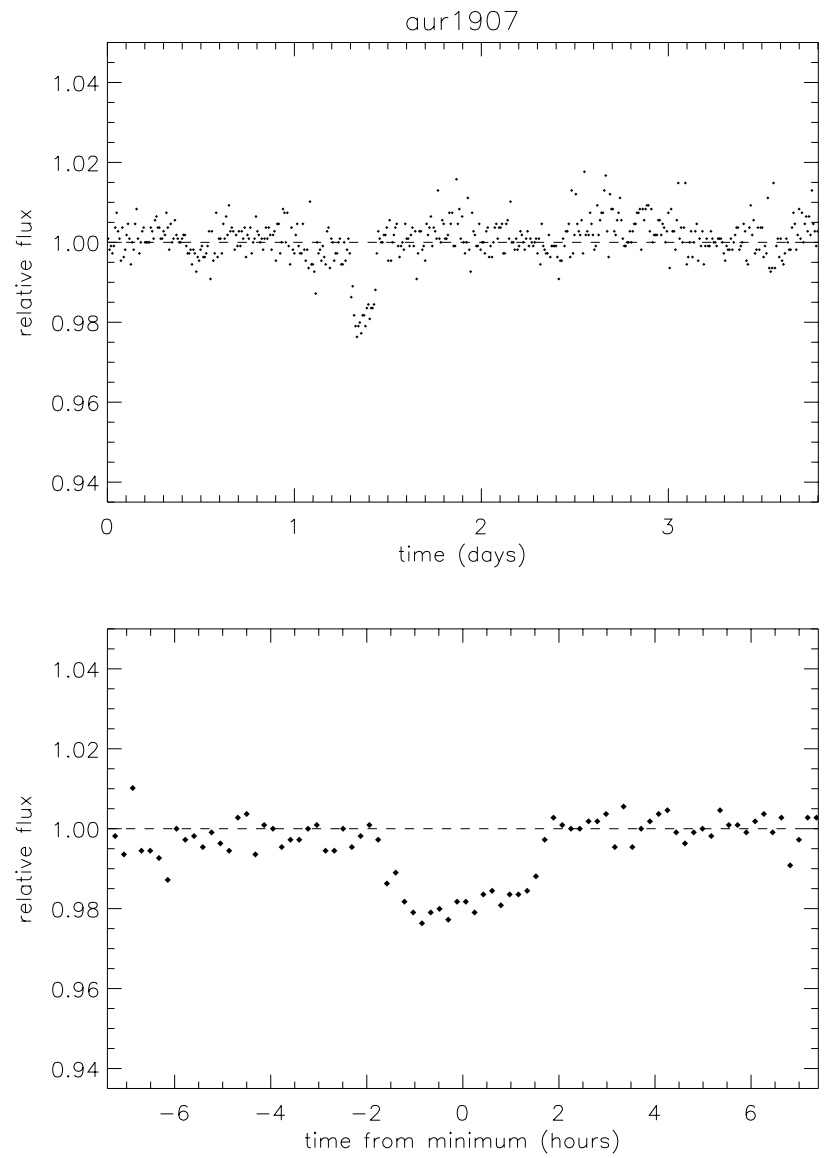

FIGURE 1. (Upper panel) PSST photometric light curve for star $1907(V=11.2, B-V=0.2)$ in Auriga, binned and phased to a period of 3.80 days. The light curve is constant outside of eclipse. (Lower panel) The same data near times of transit. The transit is flat-bottomed with a depth of $0.019 \mathrm{mag}$ and a duration of 3.7 hours, consistent with a transit of a Jovian planet across a Sun-like star.

the orbit induced by the planet. Furthermore, it is only for such bright systems that the host of follow-up measurements that are currently being pursued for HD 209458 [9] and other bright extrasolar-planet stars [10] might also be enabled.

The difficulty facing such surveys is not that of obtaining the requisite photometric precision or phase coverage, as several of the aforementioned projects have achieved these requirements. Rather, the current challenge is that of efficiently rejecting astrophysical false positives, i.e. systems containing an eclipsing binary, whose resulting photometric light curves mimic that of a Jupiter passing in front of a Sun-like star.

Brown [11] recently presented detailed estimates of rates of such false alarms for 


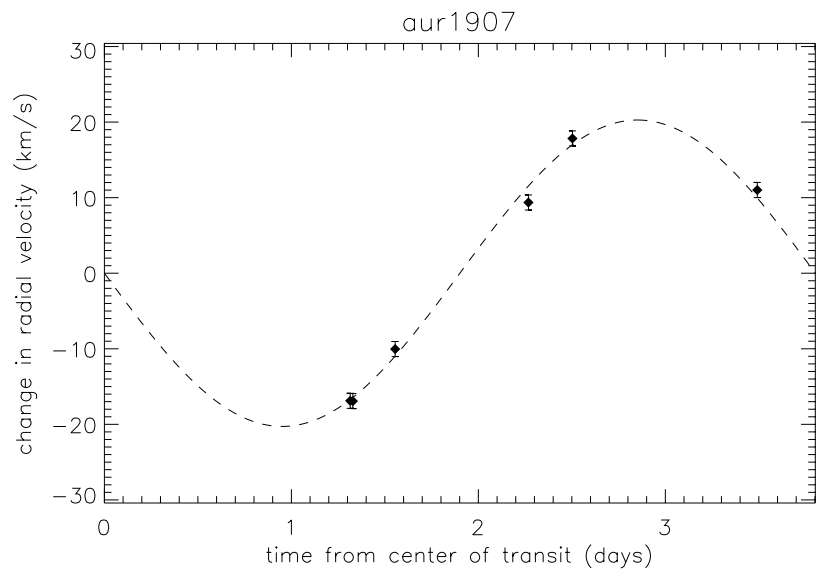

FIGURE 2. Shown are the changes in radial velocity for star 1907 in Auriga (Figure 1) as derived from high-resolution spectra gathered over 3 consecutive nights with the Palomar 60-inch echelle spectrograph. The typical precision is $1 \mathrm{~km} \mathrm{~s}^{-1}$. The observed variation is consistent with an orbit as predicted by the photometric phase and period. However, the amplitude of the radial velocity orbit is $20 \mathrm{~km} \mathrm{~s}^{-1}$, indicating a low-mass stellar companion.

these wide-field surveys. The three dominant sources of such signals are (1) grazing incidence eclipses in systems consisting of two main-sequence stars, (2) central transits of low-mass stars in front of large main-sequence stars (typically M-dwarfs passing in front of F-stars), and (3) binary systems undergoing deep eclipses, blended with the light of a third star that falls within the instrumental point-spread-function (the blending star may be either physically associated or simply lying along the line-of-sight). Brown finds that these various forms of astrophysical false positives will likely occur at a combined rate nearly 12 times that of true planetary transits. A direct means to investigate the true nature of each candidate would be high-precision radial-velocity monitoring, but this would impose a hefty burden upon the large telescopes that are required. Thus, all such transit surveys must adopt strategies for efficiently rejecting the majority of such false alarms, to bring the number of candidates to a manageable level.

\section{EXAMPLES OF ASTROPHYSICAL FALSE POSITIVES}

For the benefit of researchers pursuing such transit surveys, we present here several examples of astrophysical false positives. These candidate transit signals were identified in a survey of a $6^{\circ} \times 6^{\circ}$ field in Auriga by the PSST instrument at Lowell Observatory. A description of the instrument and the results of the survey will be presented elsewhere. Our goal here is to present the follow-up spectroscopy performed with the Palomar 60inch telescope, which demonstrates conclusively that these candidates are indeed false alarms. Access to telescopes in the range of $1-2 \mathrm{~m}$ is widely available. As a result, 

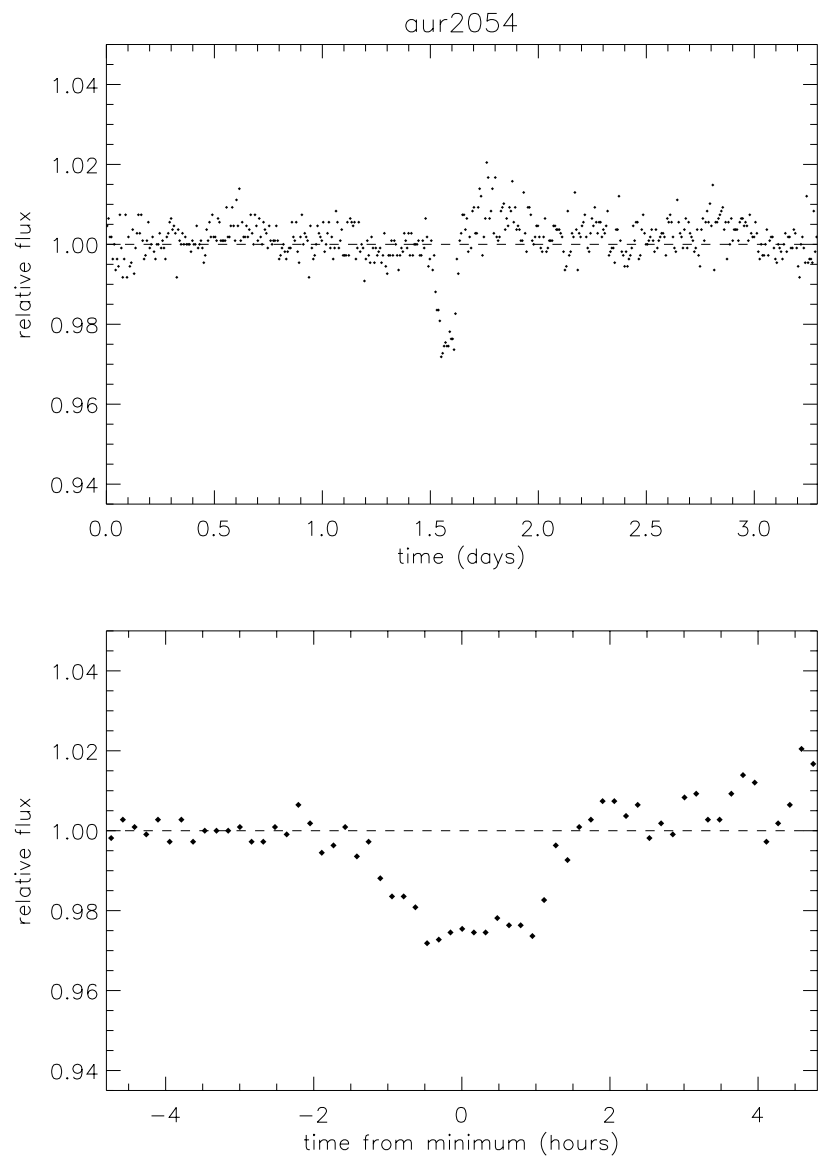

FIGURE 3. (Upper panel) PSST photometric light curve for star $2054(V=11.3, B-V=1.5)$ in Auriga, binned and phased to a period of 3.29 days. The light curve is constant outside of eclipse. (Lower panel) The same data near times of transit. The transit is flat-bottomed with a depth of 0.023 mag and a duration of 2.4 hours, consistent with a transit of a Jovian planet across a Sun-like star.

such observations are a reasonable means by which to dispose of such contaminants. Similar work to identify the true nature of candidates arising from the Vulcan survey was presented in [12].

\section{Star 1907: An M-dwarf in eclipse}

The PSST photometric light curve (Figure 1) for star $1907(V=11.2, B-V=0.2)$ revealed a flat-bottomed transit with a period of 3.80 days, a depth of 0.019 mag and 


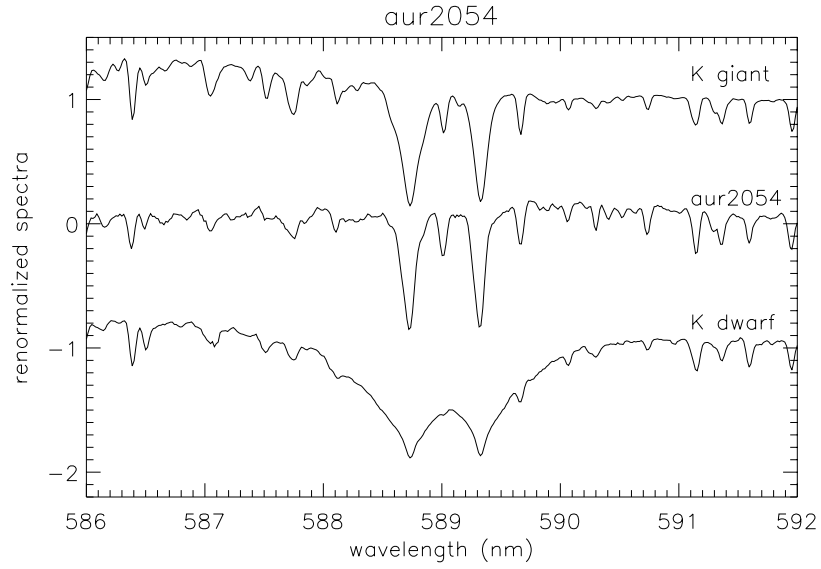

FIGURE 4. The central curve shows a Palomar 60-inch echelle spectrum of star 2054 in Auriga (Figure 3) in the region of the gravity-sensitive sodium D lines. Spectra of two K-stars with approximately the same color are also shown: The lower spectrum is that of the K5-dwarf GJ 380, demonstrating the very broad sodium lines indicative of the high gravity of a dwarf star. The upper spectrum is that of the K5.5giant HR 5200, where the sodium lines are unbroadened. The semblance of the spectrum of the candidate star to that of HR 5200 indicates that the star is a giant. Since a 3.3-day period would place the object within the physical radius of the giant, this system is likely a blend of K-giant and a fainter eclipsing binary.

a duration of 3.7 hours. The light curve is constant outside of eclipse. However, radial velocities (Figure 2) derived from high-resolution spectra gathered over 3 consecutive nights with the Palomar 60-inch echelle spectrograph (with a typical precision of $1 \mathrm{kms}^{-1}$ ) revealed an orbit consistent with the period and phase derived by the photometry, but with an amplitude of $20 \mathrm{~km} \mathrm{~s}^{-1}$. This large Doppler variation indicates a low-mass stellar companion.

\section{Star 2054: An eclipsing binary blended with a giant star}

The photometric time series (Fsigure 3) for star $2054(V=11.3, B-V=1.5)$ shows a flat-bottomed eclipse with a period of 3.29 days, a depth of $0.023 \mathrm{mag}$, and a duration of 2.4 hours. The light curve is constant outside of eclipse. Figure 4 shows a portion of the spectrum of this object gathered with the Palomar 60-inch echelle in the region of the gravity-sensitive sodium D lines. The unbroadened profile of the sodium features reveals this star to be a K-giant. Since a 3.3 day orbit about a K-giant would place it within the physical radius of the star, we must be seeing a blend of a K-giant and a fainter eclipsing binary (which may or may not be physically associated). 

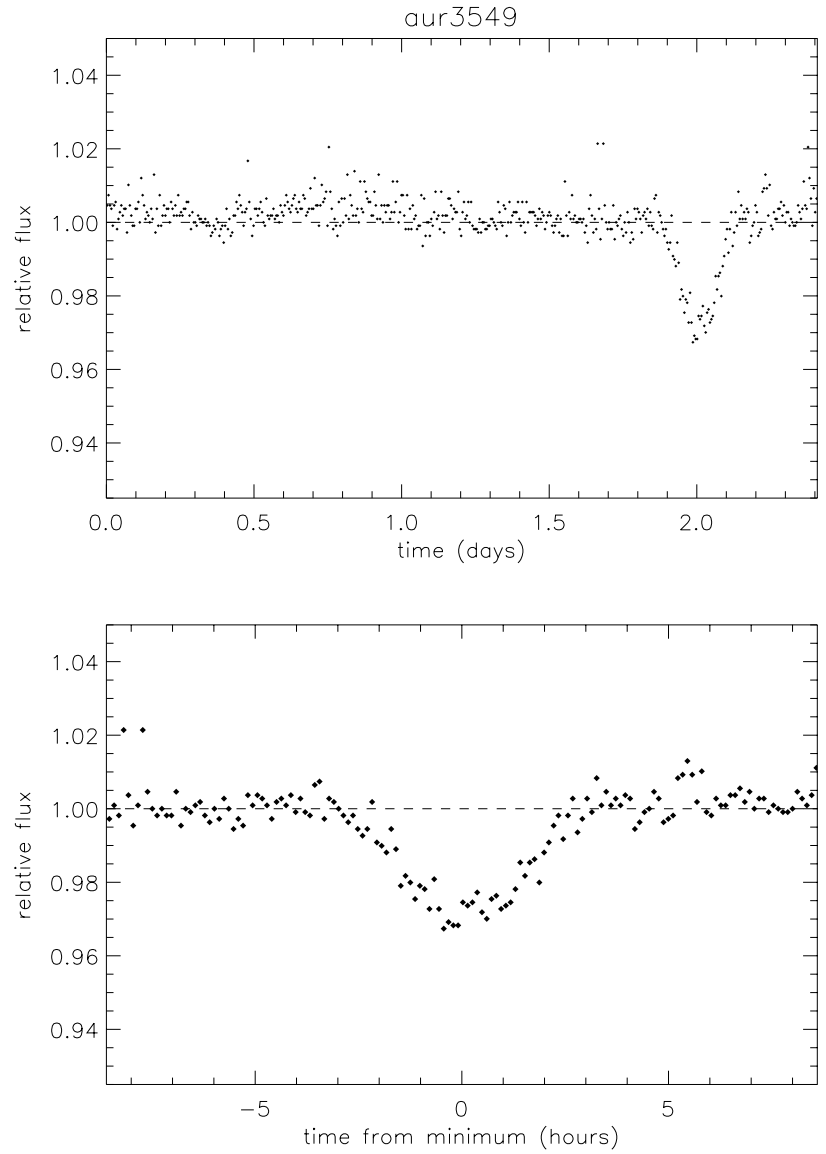

FIGURE 5. (Upper panel) PSST photometric light curve for star $3549(V=11.6, B-V=1.1)$ in Auriga, binned and phased to a period of 2.41 days. There is some evidence for a secondary eclipse in the light curve, near a time of 0.4 days. (Lower panel) The same data near times of transit. The transit is somewhat V-shaped, with a depth of 0.028 mag and a duration of 4.3 hours. Although marginally consistent with a planetary transit, these data hint at an eclipsing binary system.

\section{Star 3549: An eclipsing binary blended with an unassociated star}

The photometric light curve (Figure 5) for star $3549(V=11.6, B-V=1.1)$ shows a mildly $\mathrm{V}$-shaped eclipse with a period of 2.41 days, a depth of $0.028 \mathrm{mag}$, and a duration of 4.3 hours. Our suspicions were raised by the hint of a faint secondary eclipse in the light curve. Examination of the Digitized Sky Survey image (Figure 6) reveals two sources within the PSF of the PSST instrument. Photometry of the field during the time of a subsequent eclipse (conducted with a 14-inch telescope, which afforded a 


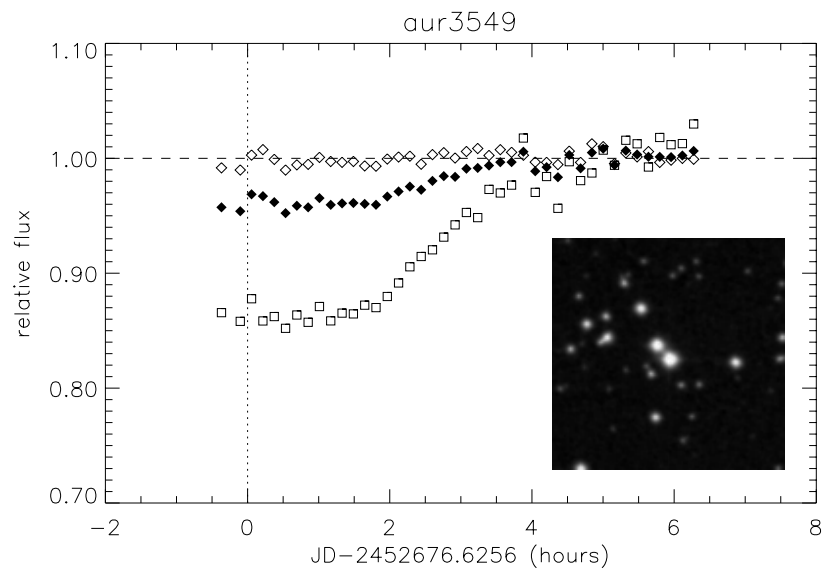

FIGURE 6. The 2 arcmin $\times 2$ arcmin Digitized Sky Survey image (inset) of star 3549 in Auriga (Figure 5) reveals two sources within the PSF of the PSST instrument. Photometry of the field during the time of a subsequent eclipse (the predicted time of center of transit is shown as $t=0$ in this figure) shows the central brighter source to be constant in time (open diamonds). The fainter star to the NE undergoes a deep (14\%) eclipse (open squares). When the light from both stars is summed in a single photometric aperture, the light curve shown with black diamonds results. This photometric time series reproduces that observed by the PSST (Figure 5). Thus, this candidate is the blend of the fainter eclipsing binary to the NE, and the brighter physically-unassociated central star.

significant increase in the angular resolution over the data gathered with the PSST) finds that the central brighter source is constant, whereas the fainter star to the NE undergoes a deep (14\%) eclipse (Figure 6). When the light from both stars in summed in a single photometric aperture, the light curve that results reproduces that observed by the PSST. Hence, this system is a blend of an eclipsing binary (the fainter star to the NE), and the physically unassociated brighter star.

\section{Star 4922: A grazing-incidence eclipsing binary}

The photometric light curve (Figure 7) for star $4922(V=12.0, B-V=0.4)$ reveals a clearly V-shaped eclipse with a period of 1.52 days, a depth of $0.021 \mathrm{mag}$, and a duration of 2.0 hours. The light curve is constant outside of eclipse, but the shape of the transit already hints at a grazing-incidence eclipsing binary. Palomar 60-inch echelle spectra (Figure 8) gathered on three consecutive nights clearly show two sets of absorption features, and nightly variations in the radial velocity of the components that are consistent with the time scale of the photometric period. The amplitude of the radial velocity orbit is approximately $90 \mathrm{~km} \mathrm{~s}^{-1}$. Combined with the $\mathrm{V}$-shaped nature of the eclipses, these data lead us to conclude that this is a grazing-incidence main-sequence binary, with a true period that is twice the photometric period. 

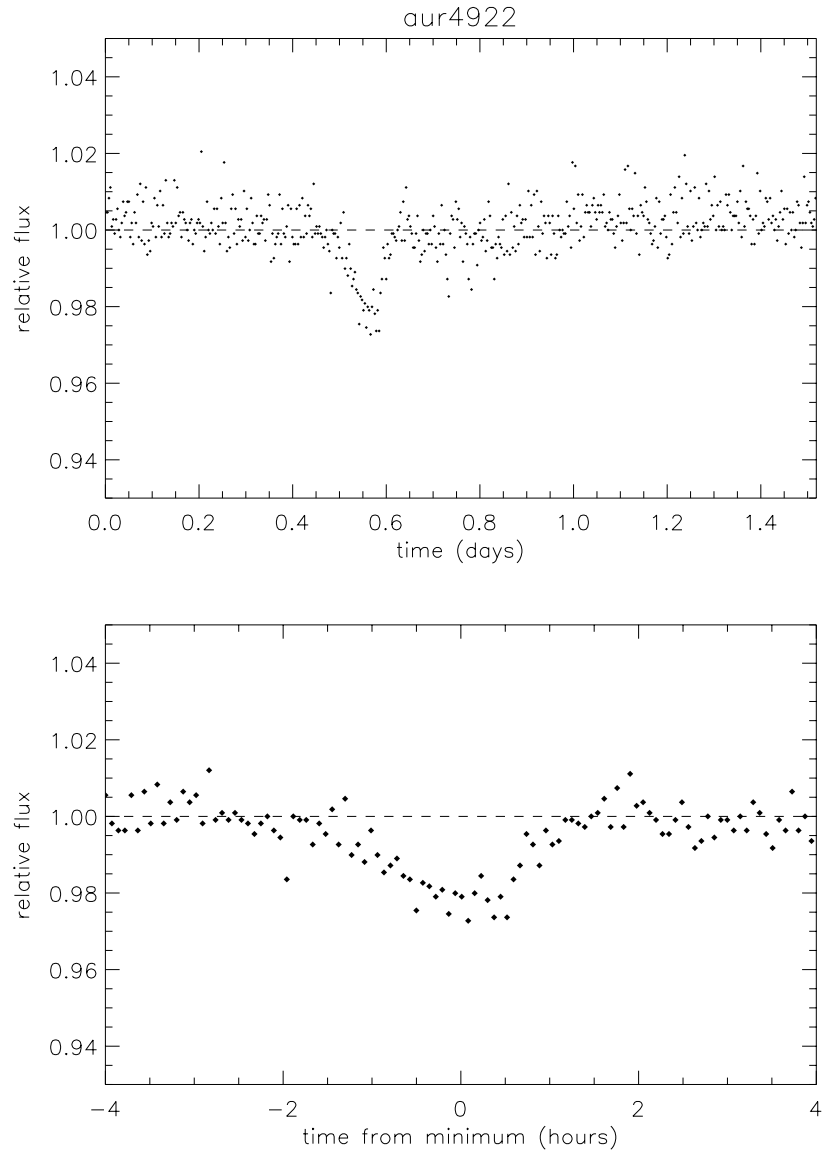

FIGURE 7. (Upper panel) PSST photometric light curve for star $4922(V=12.0, B-V=0.4)$ in Auriga, binned and phased to a period of 1.52 days. The light curve is constant outside of eclipse. (Lower panel) The same data near times of transit. The transit is V-shaped, with a depth of $0.021 \mathrm{mag}$ and a duration of 2.0 hours, indicating that the signal is likely due to a grazing-incidence binary system.

\section{EFFICIENT METHODS TO REJECT FALSE ALARMS}

The publicly-available USNO-B catalog ${ }^{7}$ [13] provides proper motions, which aid in constraining the distance of a star. Typical errors in the proper motions from the USNOB catalog are $\sim 7$ mas/yr. Objects with colors consistent with the target spectral types of $\mathrm{K}, \mathrm{G}$, or $\mathrm{F}$ and a high measured proper motion are likely dwarf stars, and thus good

${ }^{7}$ http://www.nofs.navy.mil/projects/pmm/catalogs.html 


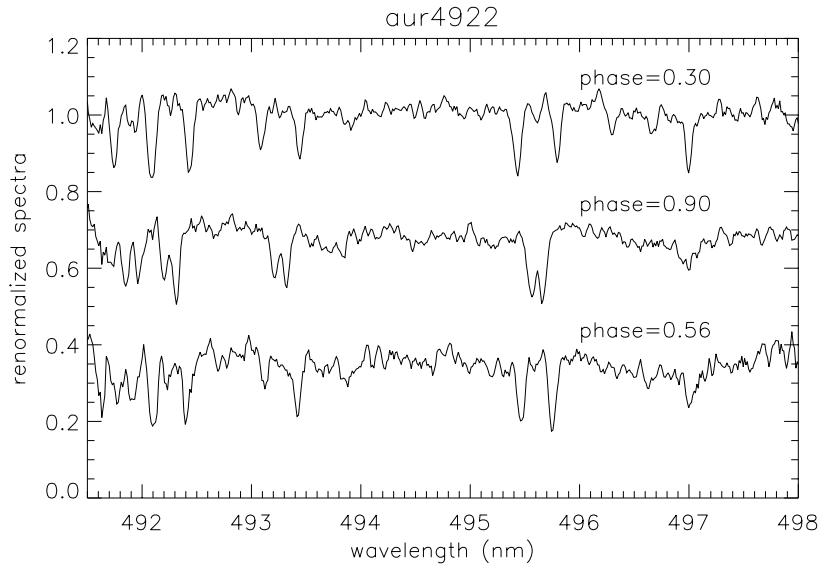

FIGURE 8. Shown are Palomar 60-inch echelle spectra of star 4922 in Auriga (Figure 7) gathered on three consecutive nights. The spectra are clearly double-lined. The variations in radial velocity are consistent with the time scale of the photometric period (the spectra are labeled by the orbital phase predicted by the photometry). The radial velocity orbit is approximately $90 \mathrm{~km} \mathrm{~s}^{-1}$. Combined with the $\mathrm{V}$-shaped nature of the eclipses, we conclude that this is likely a grazing-incidence binary, with a true period that is twice the photometric period.

candidates. Unfortunately, small proper motions are inconclusive: These stars could either be distant (hence giants), or nearby dwarfs. In addition, colors from the 2MASS catalog $^{8}$ aid in separating dwarf stars from giants. Brown [11] illustrates that the $J-K$ colors from the 2MASS catalog provide a useful discriminant: Stars with $J-K \geq 0.5$ are predominantly giants, whereas those with $J-K \leq 0.35$ are almost exclusively dwarfs (Figure 9). The target stars of wide-field transit surveys are those with radii less than $1.3 R_{\odot}$. The infrared colors of such stars extend into the intermediate region, so that the $J-K$ color is not a definitive test. Nonetheless, candidates with very red colors $(J-K>0.7)$ are almost certainly giants and can be rejected.

While the spectroscopy described in the previous section provides a reliable means to identify astrophysical false positives, it is labor- and resource-intensive. In order to more efficiently reject such contaminants, L. Kotredes and D. Charbonneau are currently assembling Sherlock, an automated follow-up telescope for wide-field transit searches [14]. Working from a list of transit candidates, Sherlock will calculate future times of eclipse, and conduct multi-color photometry of these targets, with an angular resolution that is significantly improved over the survey instruments. The large majority of false alarms should yield eclipse signals that are color-dependent, whereas planetary transits should be nearly color-independent (except for the minor effects due to limb-darkening). Sherlock will be deployed at Palomar Observatory in early 2004, and will be available to follow up candidates identified by any of the wide-field transit searches.

${ }^{8}$ http://www.ipac.caltech.edu/2mass/releases/allsky/ 


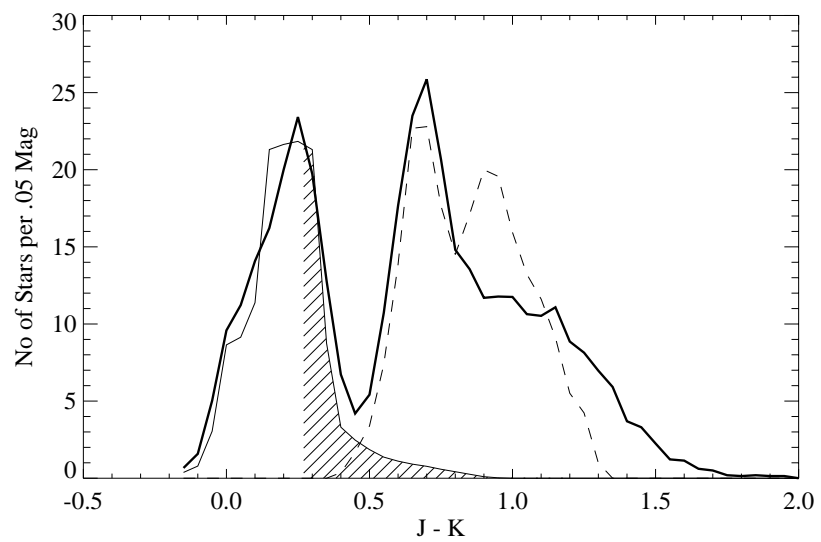

FIGURE 9. Taken from [11], courtesy T. M. Brown. The thick curve shows the histogram of star density vs $J-K$ color from the 2MASS catalog for stars observed by a STARE in a field in Cygnus. The thin solid curve shows the model used by Brown [11] to predict the occurence of main-sequence stars, and the dashed curve shows that for giants. The hatched region shows stars with radii less than $1.3 R_{\odot}$. Thus, the $J-K$ color appears to be a useful discriminant against giant stars.

\section{REFERENCES}

1. Horne, K. 2003, ASP Conf. Ser. 294: Scientific Frontiers in Research on Extrasolar Planets, eds. D. Deming \& S. Seager (San Francisco: ASP), 361, astro-ph/0301249

2. Charbonneau, D. 2003a, Space Science Reviews: ISSI Workshop on Planetary Systems and Planets in Systems, ed. S. Udry, W. Benz, \& R. von Steiger (Dordrecht: Kluwer), astro-ph/0302216

3. Brown, T. M. \& Charbonneau, D. 2000, ASP Conf. Ser. 219: Disks, Planetesimals, and Planets, eds. F. Garzon and T. J. Mahoney (San Francisco: ASP), 584, astro-ph/0005009

4. O'Donovan, F. T., Charbonneau, D., \& Kotredes, L. 2003, AIP Conf. Proc.: The Search for Other Worlds, eds. S. S. Holt \& D. Deming, astro-ph/0312289

5. Borucki, W. J., Caldwell, D., Koch, D. G., Webster, L. D., Jenkins, J. M., Ninkov, Z., \& Showen, R. 2001, PASP, 113, 439

6. Jenkins, J. M., Caldwell, D. A., \& Borucki, W. J. 2002, ApJ, 564, 495

7. Bakos, G. Á., Lázár, J., Papp, I., Sári, P., \& Green, E. M. 2002, PASP, 114, 974

8. Street, R. A. et al. 2003, ASP Conf. Ser. 294: Scientific Frontiers in Research on Extrasolar Planets, eds. D. Deming \& S. Seager (San Francisco: ASP), 405, astro-ph/0208233

9. Charbonneau, D. 2003b, ASP Conf. Ser. 294: Scientific Frontiers in Research on Extrasolar Planets, eds. D. Deming \& S. Seager (San Francisco: ASP), 449, astro-ph/0209517

10. Charbonneau, D. 2003c, ASP Conf. Ser.: IAU Symp. 219: Stars as Suns: Activity, Evolution, and Planets, eds. A. O. Benz and A. K. Dupree (San Francisco: ASP), astro-ph/0312252

11. Brown, T. M. 2003, ApJ, 593, L125

12. Latham, D. W. 2003, ASP Conf. Ser. 294: Scientific Frontiers in Research on Extrasolar Planets, eds. D. Deming \& S. Seager (San Francisco: ASP), 409

13. Monet, D. G. 2003, AJ, 125, 984

14. Kotredes, L., Charbonneau, D., Looper, D. L., \& O’Donovan, F. T. 2003, AIP Conf. Proc.: The Search for Other Worlds, eds. S. S. Holt \& D. Deming, astro-ph/0312432 